\title{
ESTIMATION OF THE POLICY EFFECTS USING COMPUTABLE GENERAL EQUILIBRIUM MODEL (THE CASE OF GEORGIA)
}

\author{
IURI ANANIASHVILI \\ Doctor of Economic Sciences, Professor \\ Ivane Javakhishvili Tbilisi State University, \\ Academician of the Georgian Academy of Economic Sciences, Georgia \\ luri.ananiashvili@tsu.ge
}

\author{
NATIA MATSIASHVILI \\ PhD Student \\ Ivane Javakhishvili Tbilisi State University, Georgia \\ natiamatsiashvili@gmail.com
}

\begin{abstract}
The findings presented in this article are to some extent continuation of the previous studies done by Georgian authors on the economy of Georgia using CGE models. The main instrument used in our research is based on a standard model tailored by Hosoe (Hosoe, 2004) to specifics of open small economy and is its modification of a certain kind. Two types of factors of production are used in this model to produce goods and services: labour and capital. The model also includes four types of commodities and production activities: agriculture, industry, construction and services. Under the necessary assumptions and restrictions, our model is a system composed of 99 equations and as many of endogenous variables. 36 of these equations correspond to the peculiarities of domestic production; 13 of them describe the government behavior; 4 characterize the investment demand function; The other 46 equations represent the behavioral characteristics of government, households, and the external sector.

The social accounting matrix corresponding to the state of the economy of Georgia in 2019 is specially constructed for our research. To build it, we used the information contained in the Geostat Supply and Use tables (SUT), separate aggregates of national accounts, balance of payments, consolidated budget, and labor force survey data.

Parameters of CGE model are calibrated based on the social accounting matrix. For this purpose, 2019 is considered as a base year and it is assumed that at that time economy of Georgia was in equilibrium. To define units of measurement for commodities and factors of production we set all prices of initial equilibrium to be equal to 1 .

Using our estimated model we arrange three different scenarios for the Georgian economy to find out what could have happened after implementing certain economic policies under ceteris paribus.

According to the first scenario, the initial equilibrium of the economy is determined as of 2019 and the rate of indirect taxes is reduced by $10 \%$. In the second scenario, we assume that the Georgian lari, has depreciated by $15 \%$ against the US dollar under ceteris paribus and we examine the expected results of the event. Lastly, in the third scenario, we assume under ceteris paribus that export increases by $20 \%$ and determine the aftereffects of export incentives.
\end{abstract}

KEYWORDS: COMPUTABLE GENERAL EQUILIBRIUM MODEL, SOCIAL ACCOUNTING MATRIX, CALIBRATION METHOD, EFFECTS OF ECONOMIC POLICY, SIMULATION.

For citation: Ananiashvili, I., Matsiashvili, N. (2021). Estimation of the Policy Effects using Computable General Equilibrium Model (The Case of Georgia). Globalization and Business. 12, 133-142. (In Georgian). https://doi.org/10.35945/gb.2021.12.018 


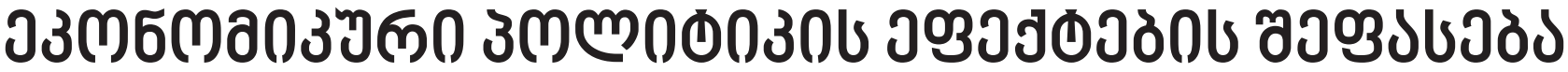

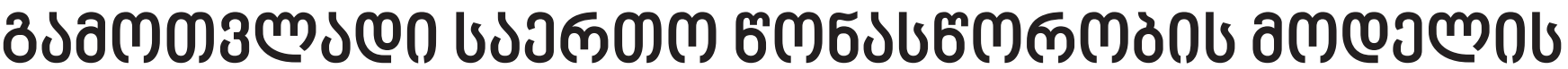

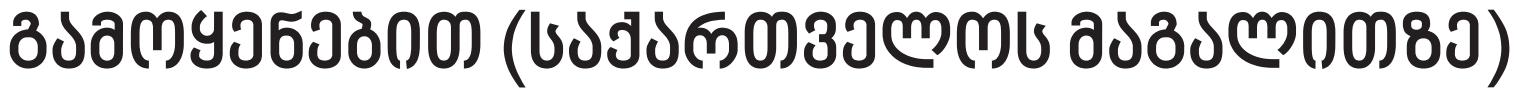

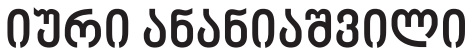

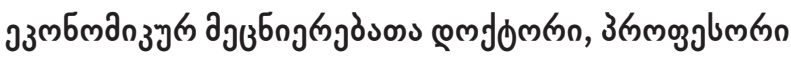

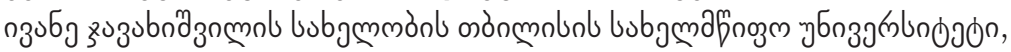

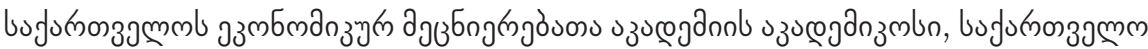 \\ luri.ananiashvili@tsu.ge
}

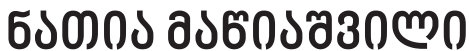

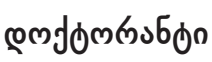

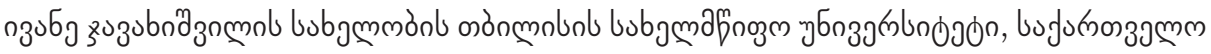

natiamatsiashvili@gmail.com

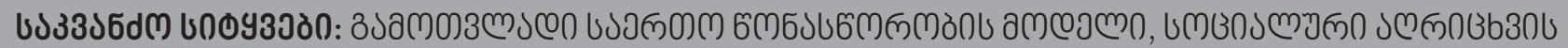

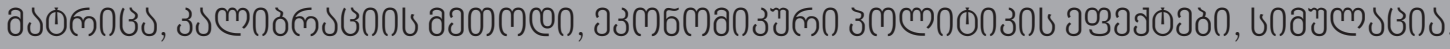

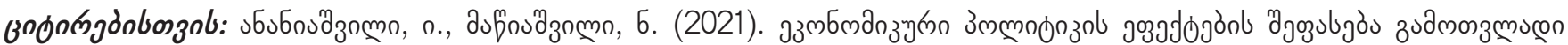

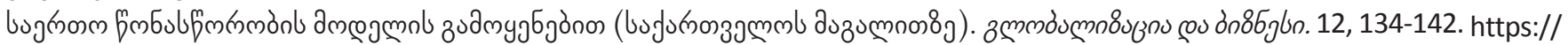
doi.org/10.35945/gb.2021.12.018

\section{әอઢ১З১ल00}

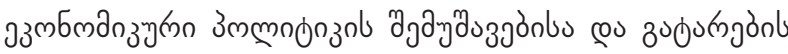

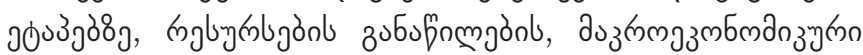

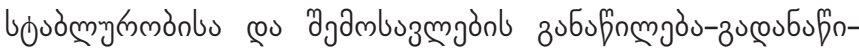

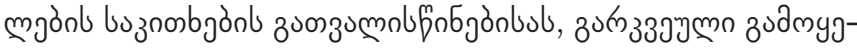

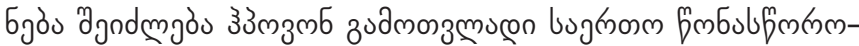

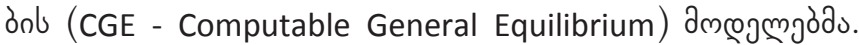
s ऊl

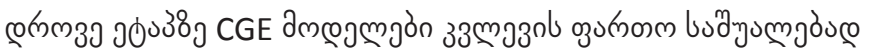

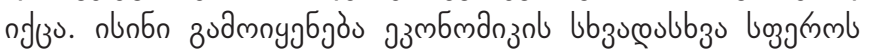

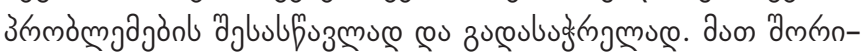

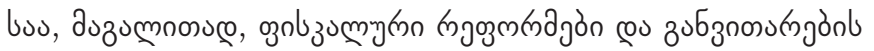
उmmnonzo (asz., Perry, 2003; Willem Gunning \& Keyzer,

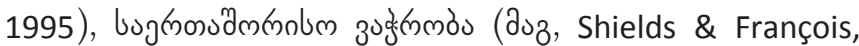

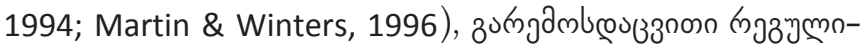
ŕjôn งbumno8n ( Duz., Weyant, 1999; Bovenberg, 1996;

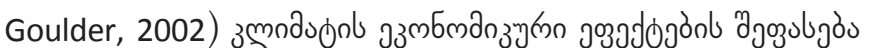

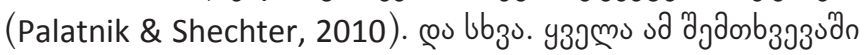

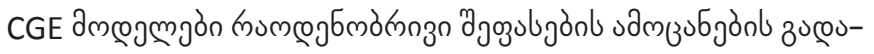

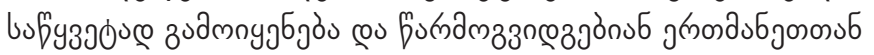

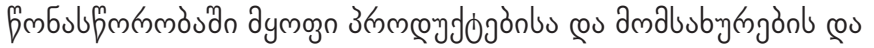

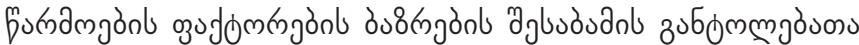
unbojan bubnon.

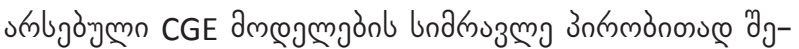

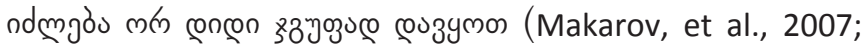

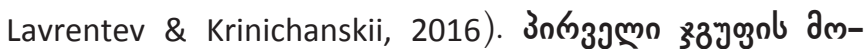

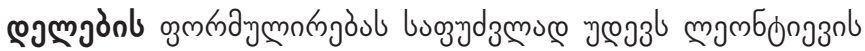

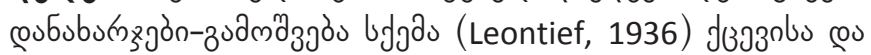

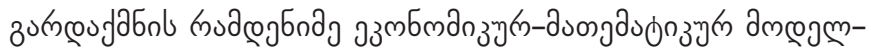

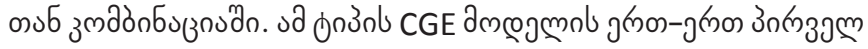

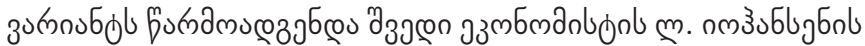

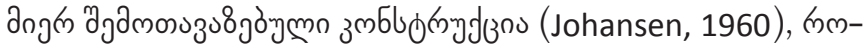

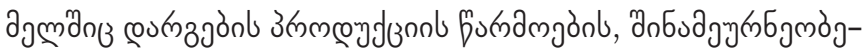

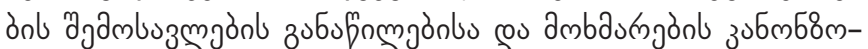

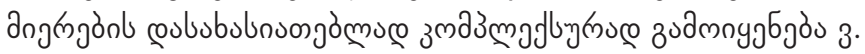

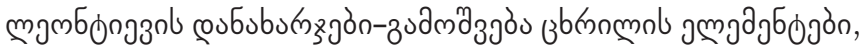

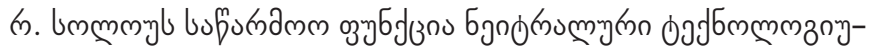

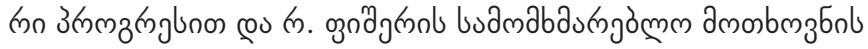

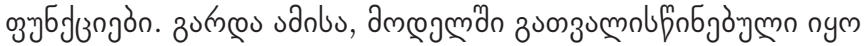

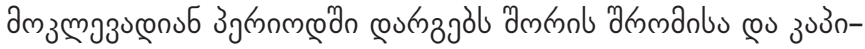

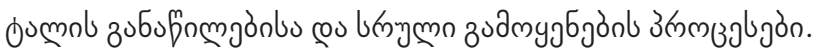

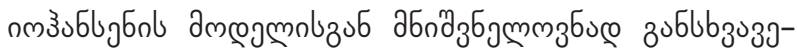

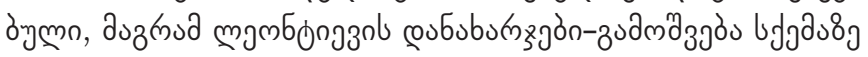

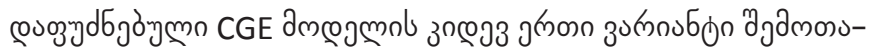

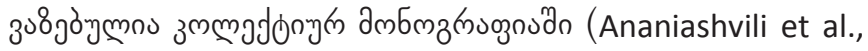

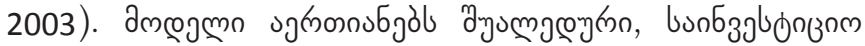

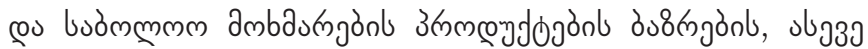

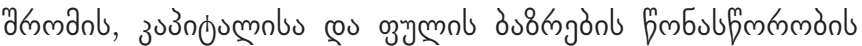
забомm 


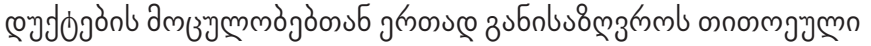

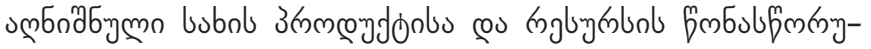

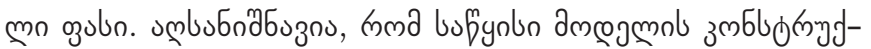

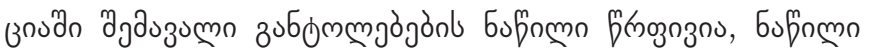

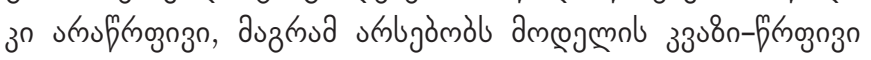

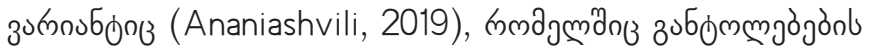

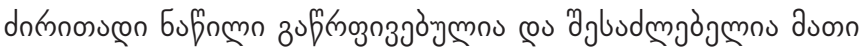

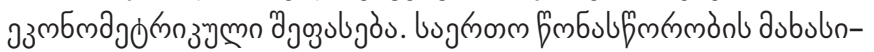

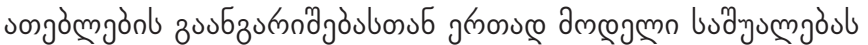

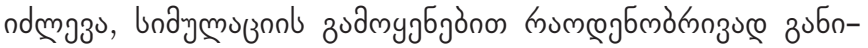
bus@z

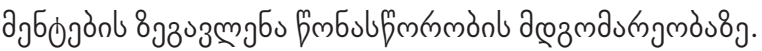

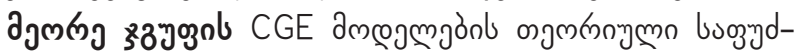

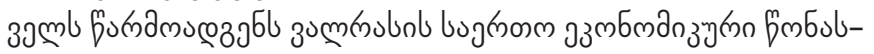

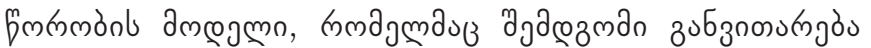

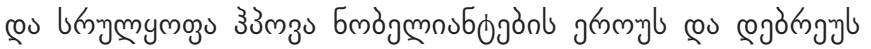

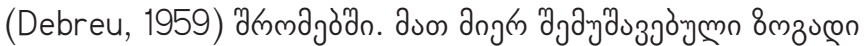

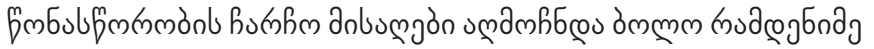

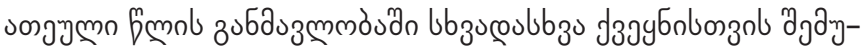

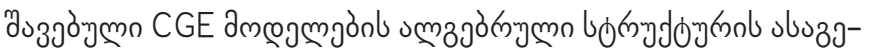

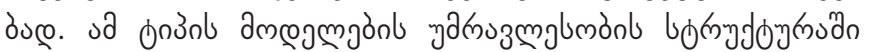

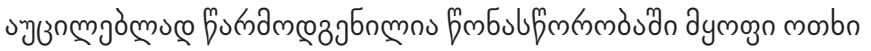

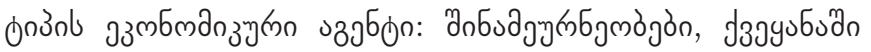

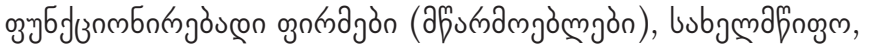

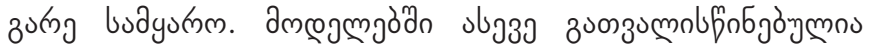

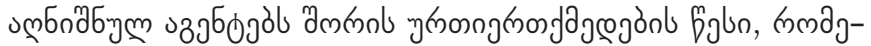

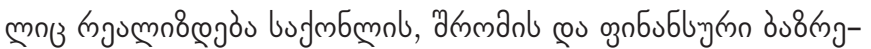

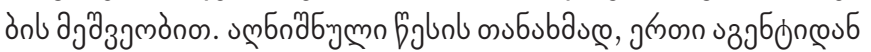

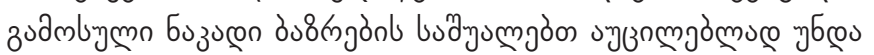

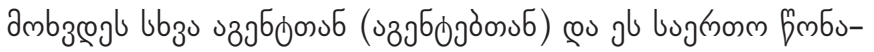

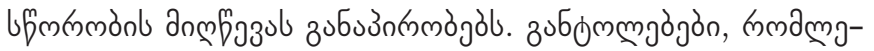

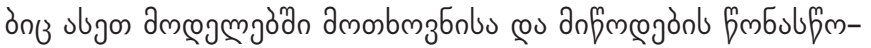

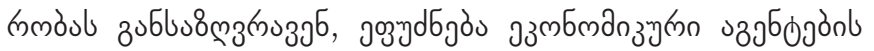

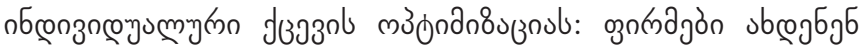

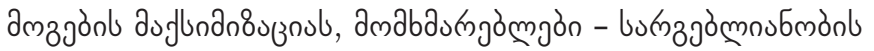
auflonan8oznol.

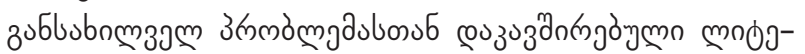

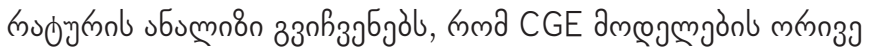

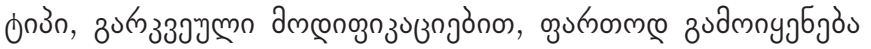

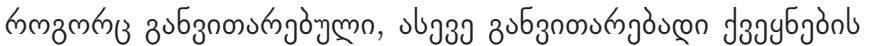

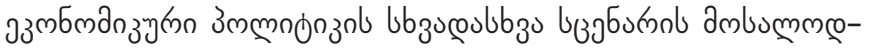

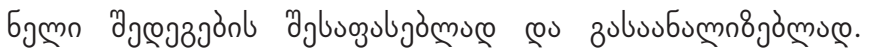

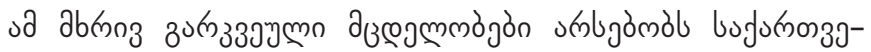

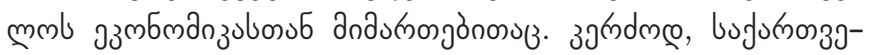

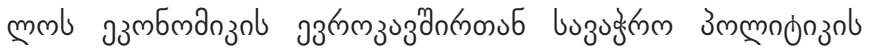

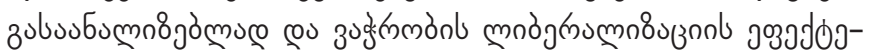

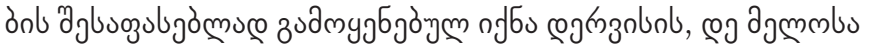

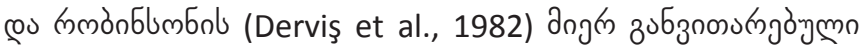

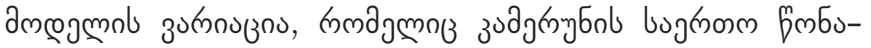

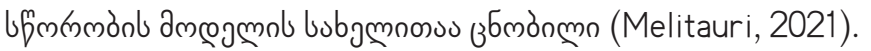

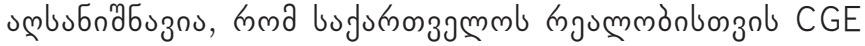

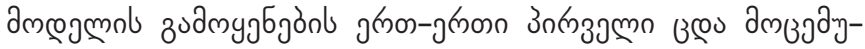

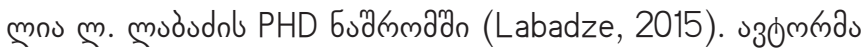

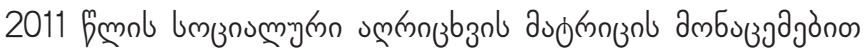

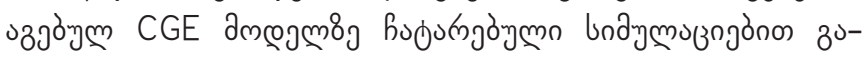

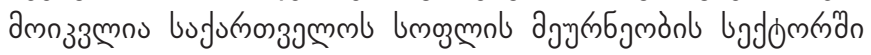

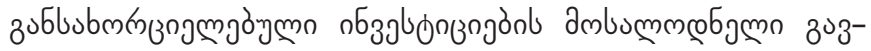

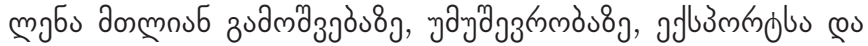

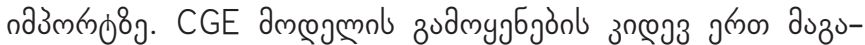

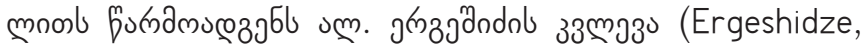

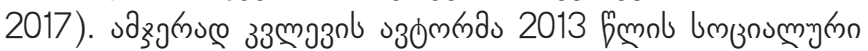

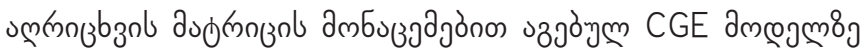

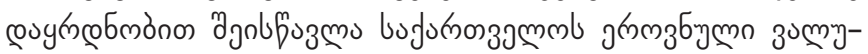

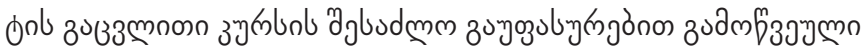

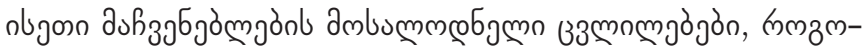

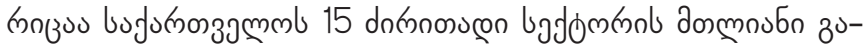

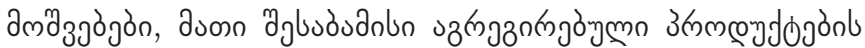

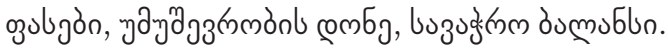

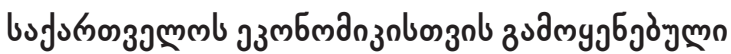

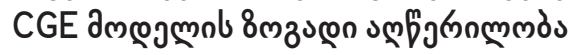

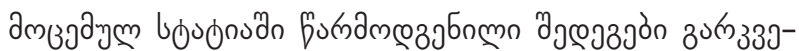

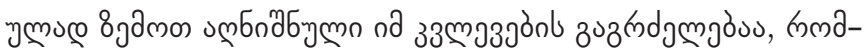

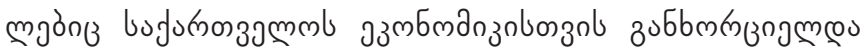

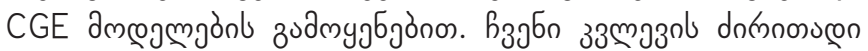

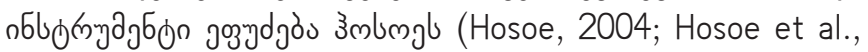
2010) dng क

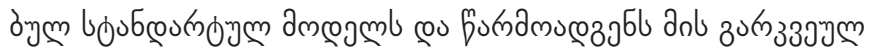

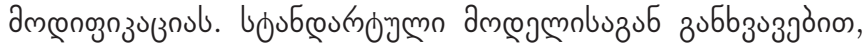

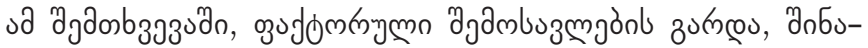

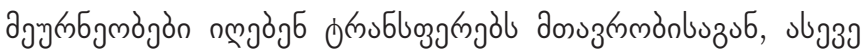

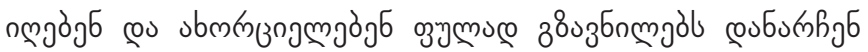

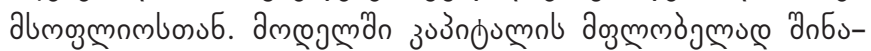

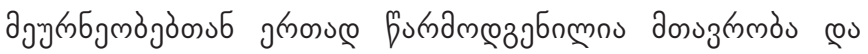
coubuknghbn alumoुmam.

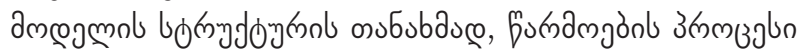

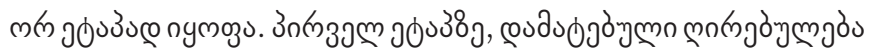

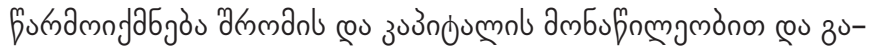

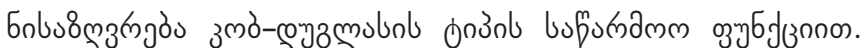

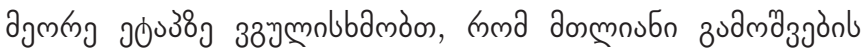

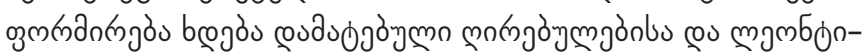

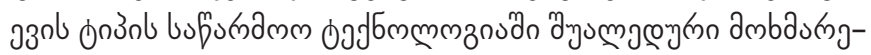

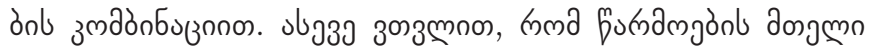

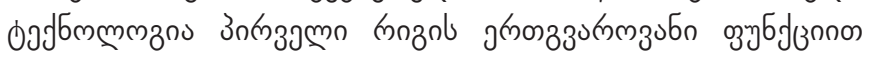

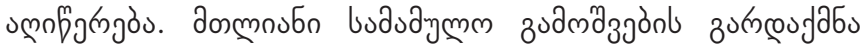

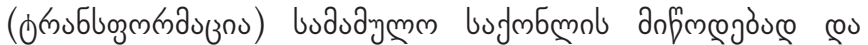

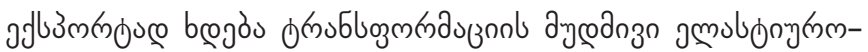
on b (Constant Elasticity of Transformation - CET) djmbg 


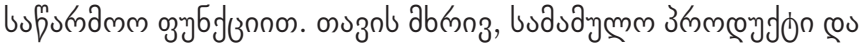

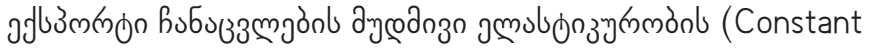
Elasticity of Substitution - CES) oुjø

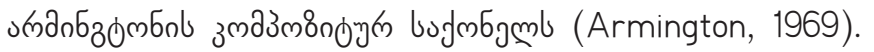

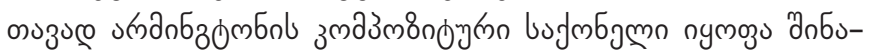

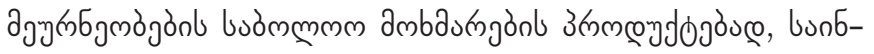

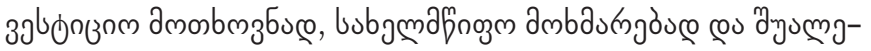

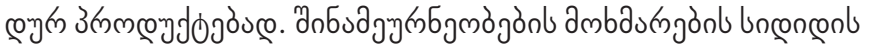

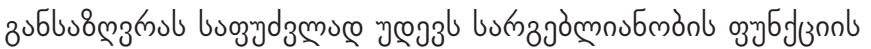

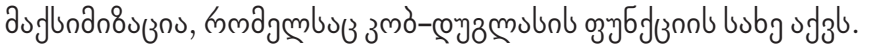

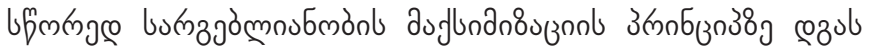

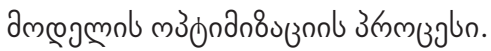

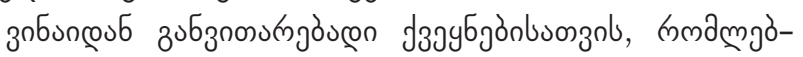

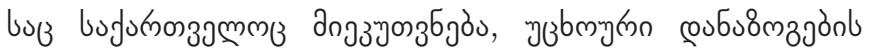

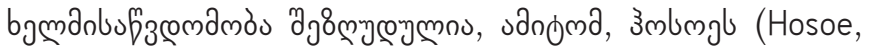

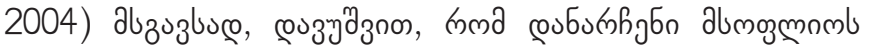

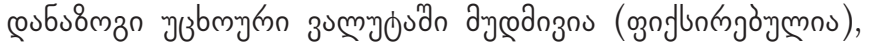

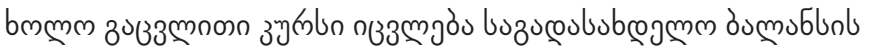

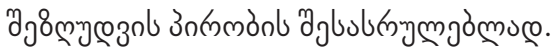

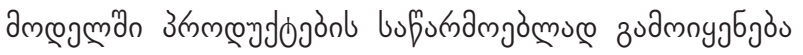

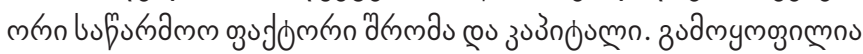

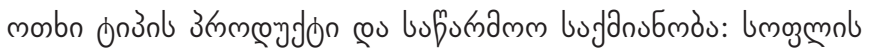

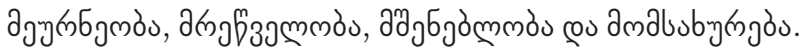

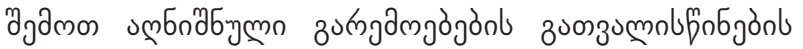

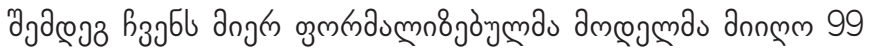

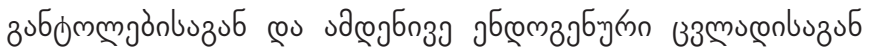

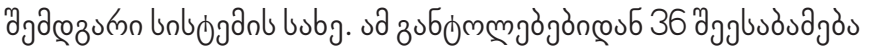

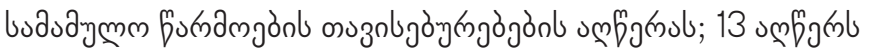

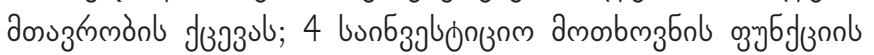

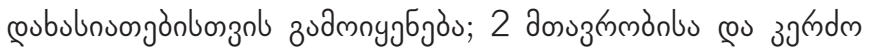

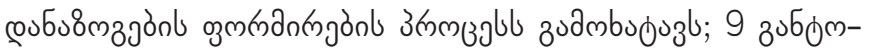

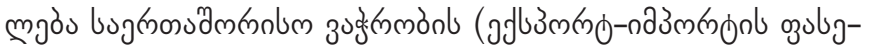

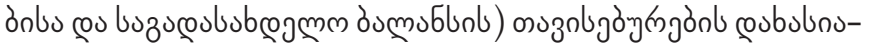

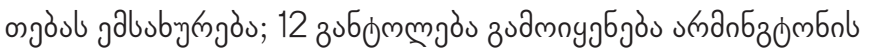

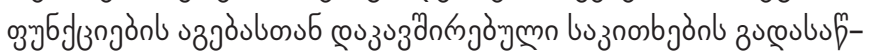

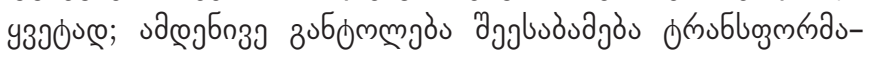

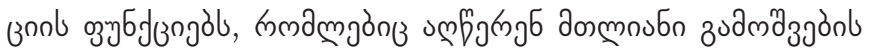

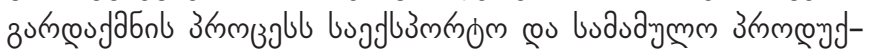

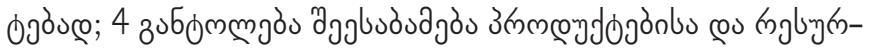
b

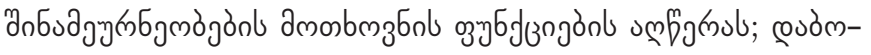

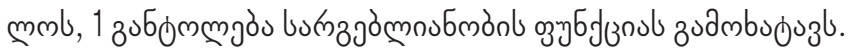

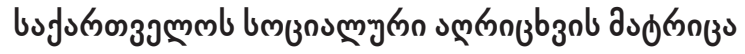

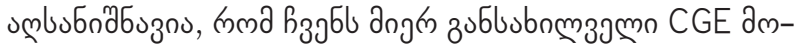

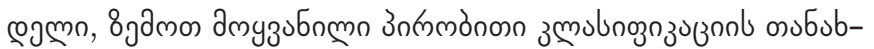

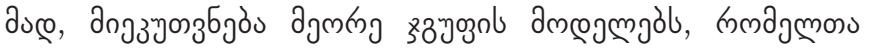

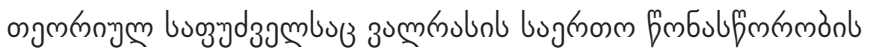

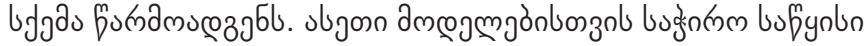

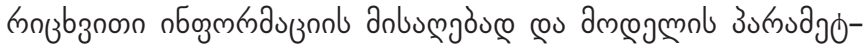

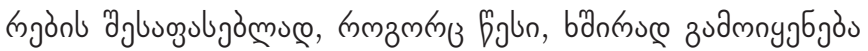

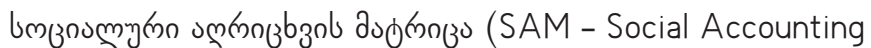

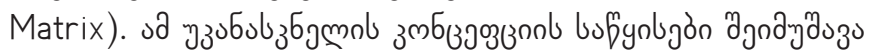

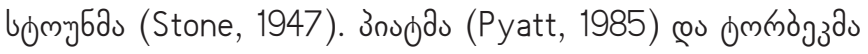

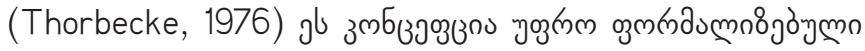

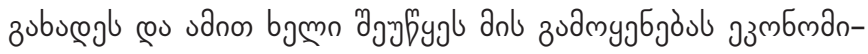

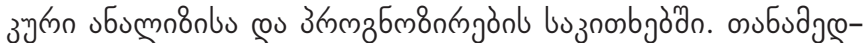

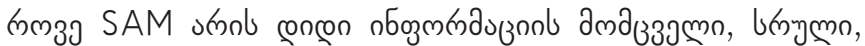

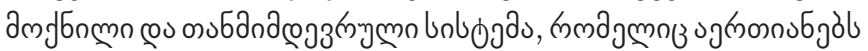

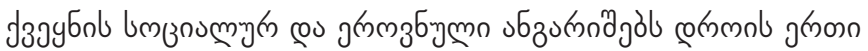

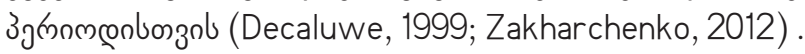

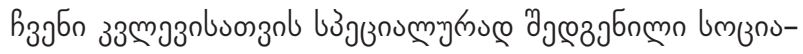

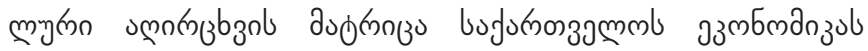

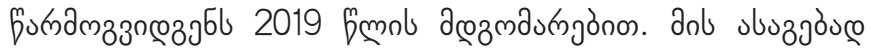

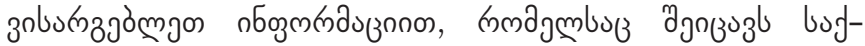

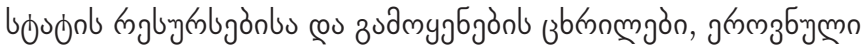

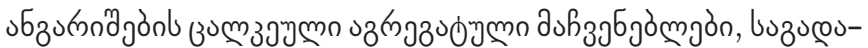

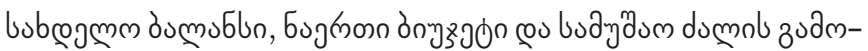

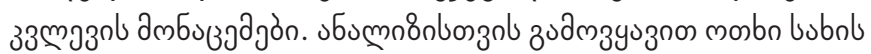

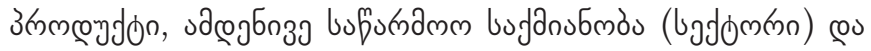

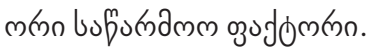

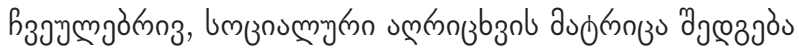

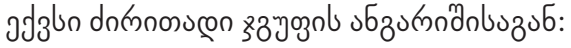

- bujanubmós gos उmmcoidon;

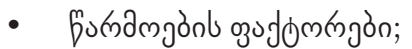

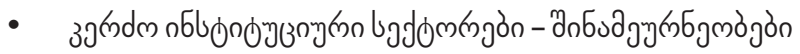
cou buagpurnagm badom non;

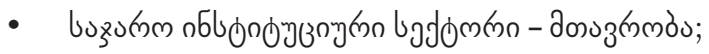

- zuzoducomol ubzumogno;

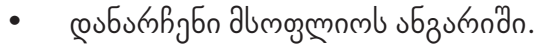

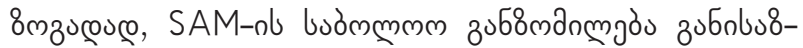

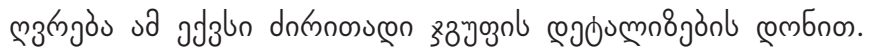

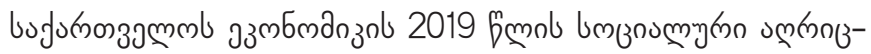

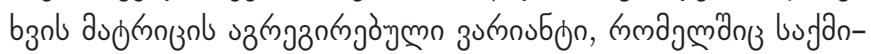

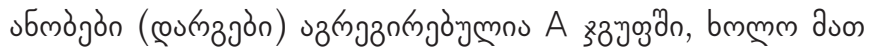

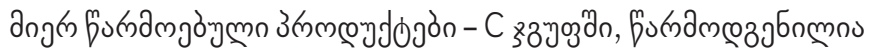

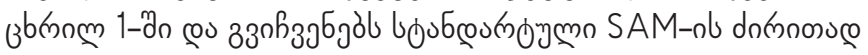

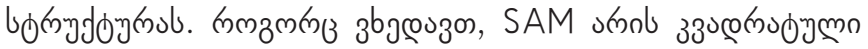

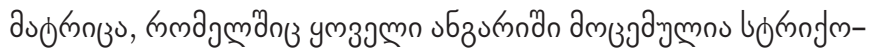

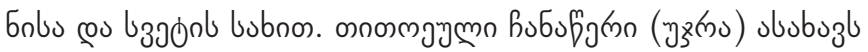

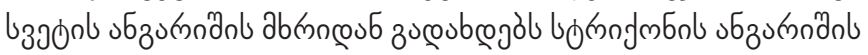

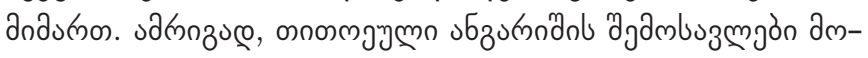

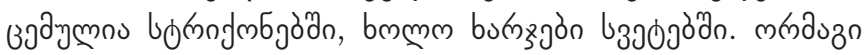

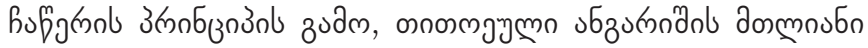

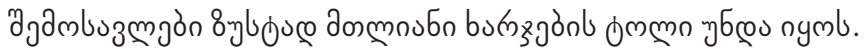

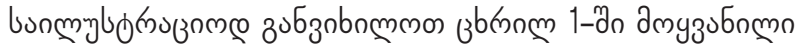

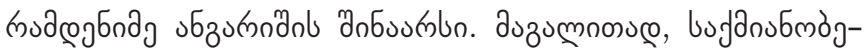




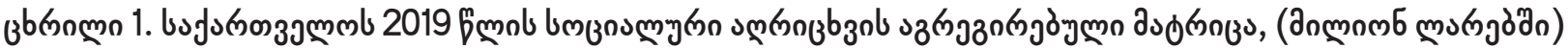

\begin{tabular}{|c|c|c|c|c|c|c|c|c|c|c|}
\hline & A & C & TTM & $F$ & $\mathrm{H}$ & G & $\mathrm{T}$ & $(S-1)$ & RoW & byem \\
\hline A & & 78562,9 & & & & & & & & 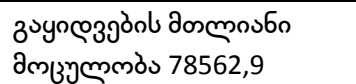 \\
\hline C & 35425,1 & & 12421,6 & & 34748,8 & 6457,7 & & 12460,4 & 26999,6 & 128513,1 \\
\hline TTM & & 12421,6 & & & & & & & & 12421,6 \\
\hline$F$ & 43137,8 & & & & & & & & & 43137,8 \\
\hline $\mathrm{H}$ & & & & 39146,9 & & 4374,1 & & & 4886,5 & 48407,5 \\
\hline G & & & & 1065,8 & & & 11417,8 & & 423,6 & 12907,2 \\
\hline $\mathrm{T}$ & & 6114,9 & & & 5302,9 & & & & & 11417,8 \\
\hline S-I & & & & & 7686,4 & 2075,4 & & & 2698,5 & 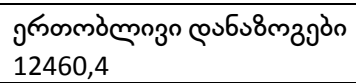 \\
\hline RoW & & 31413,8 & & 2925,1 & 669,3 & & & & & 35008,2 \\
\hline bym & 78562,9 & 128513,1 & 12421,6 & 43137,8 & 48407,5 & 12907,2 & 11417,8 & 12460,4 & 35008,2 & 382836,4 \\
\hline
\end{tabular}

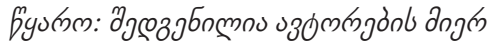

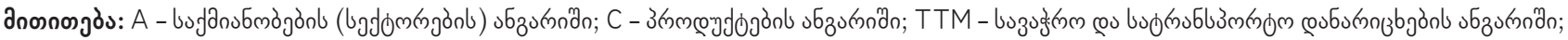

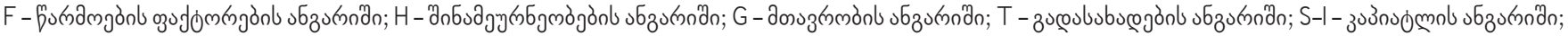

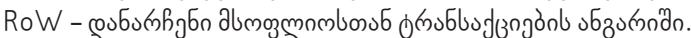

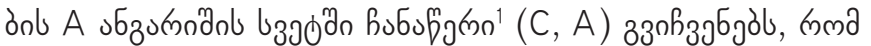

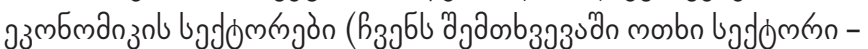

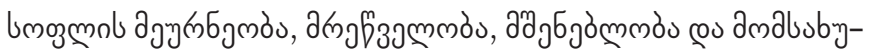

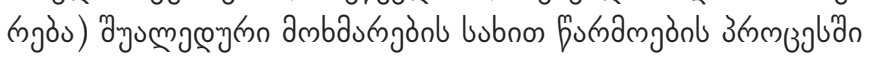

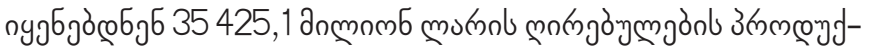

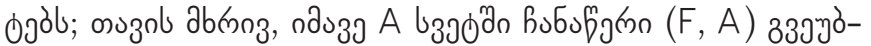

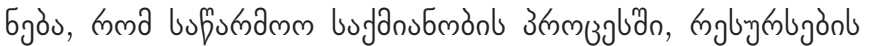

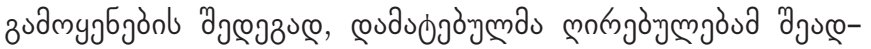

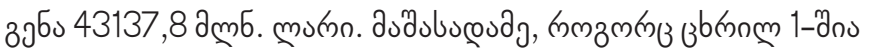

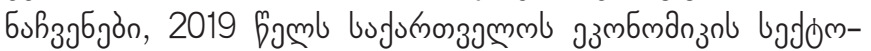

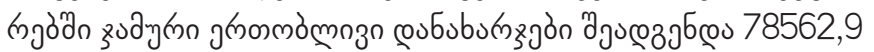
amb. mukb.

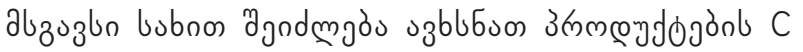

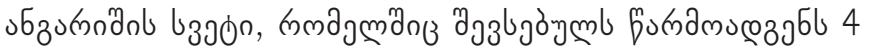

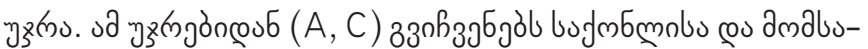

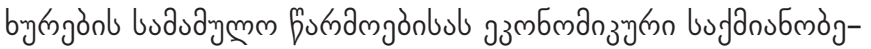

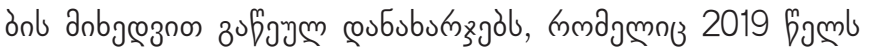

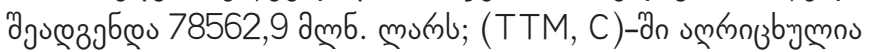

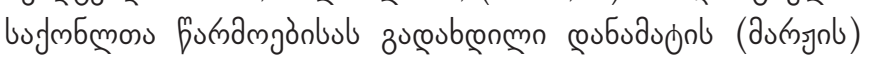

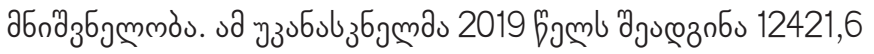

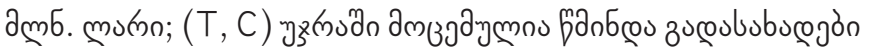

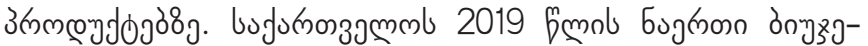

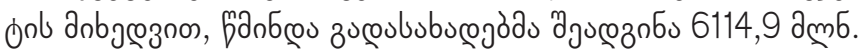

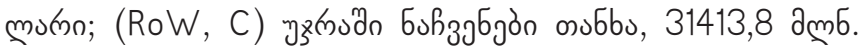

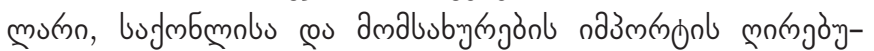

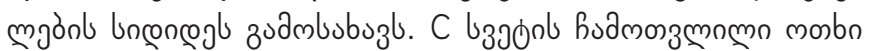

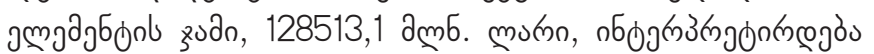

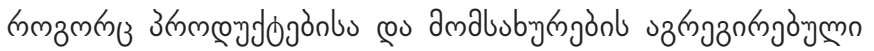

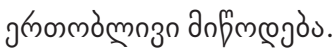

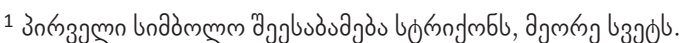

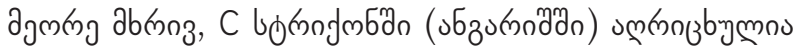

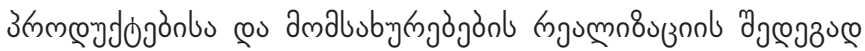

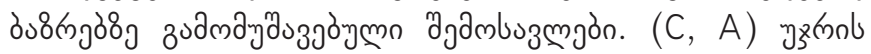

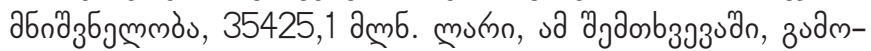

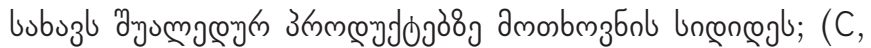

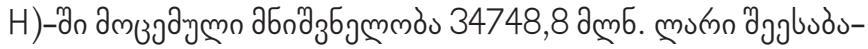

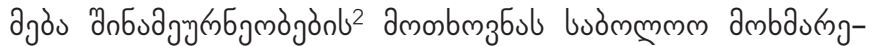

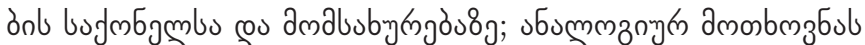

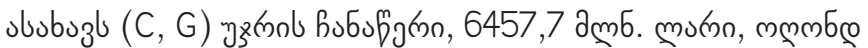

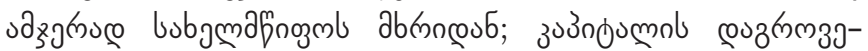

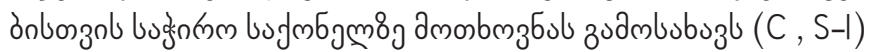

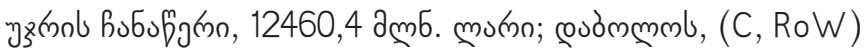

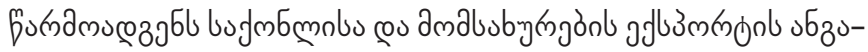

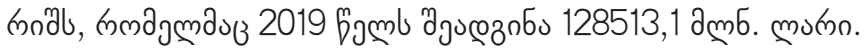

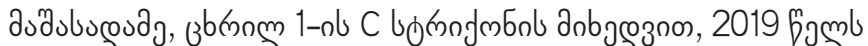

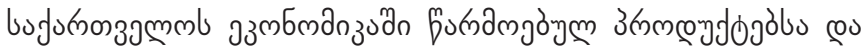

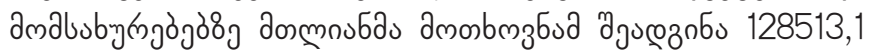
amb. mumn.

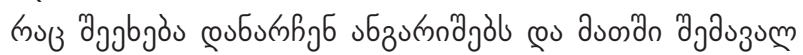

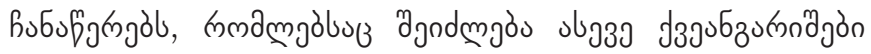

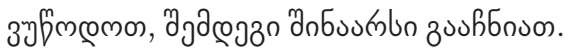

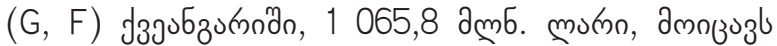

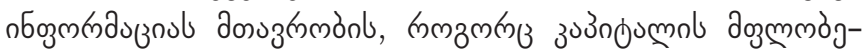

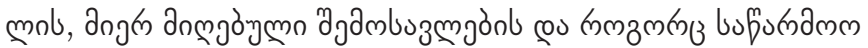

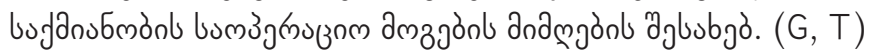

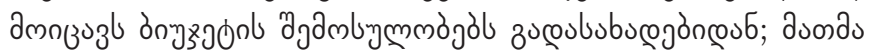

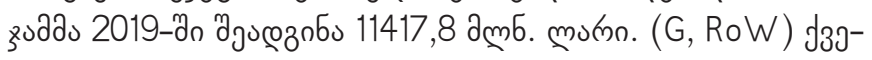

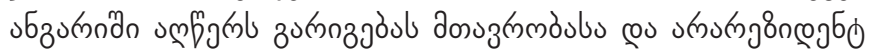

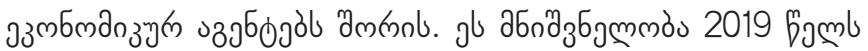

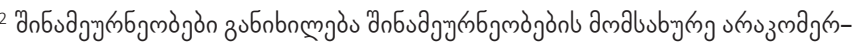

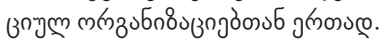




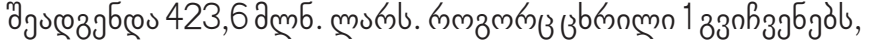

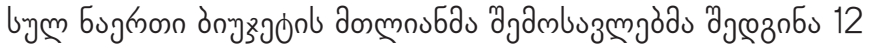
907,2 2 $\mathrm{m} 6$ mumn.

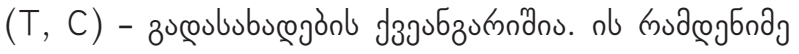

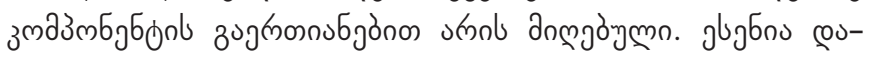

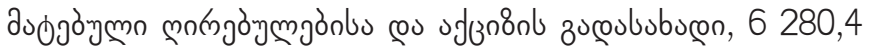

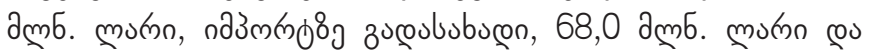

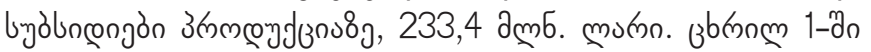

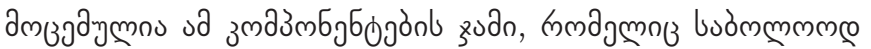

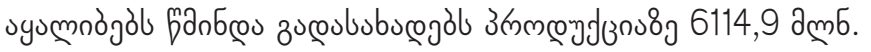

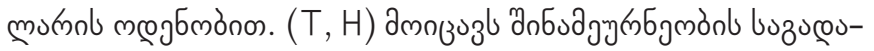

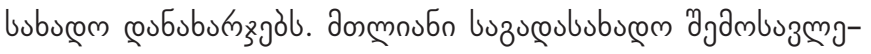

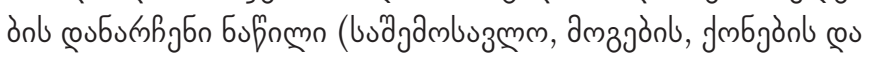

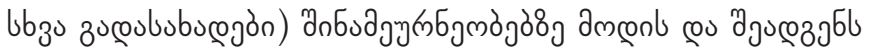
5302,9 am5. mukrl.

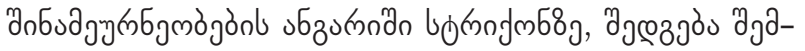

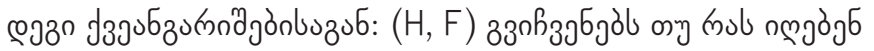

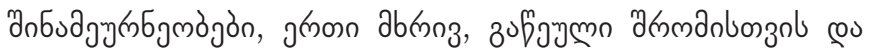

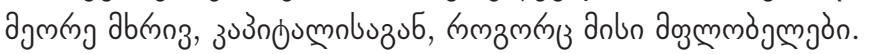

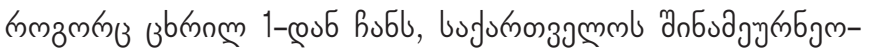

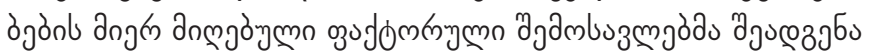

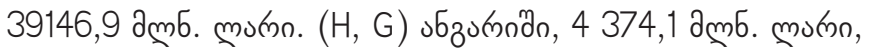

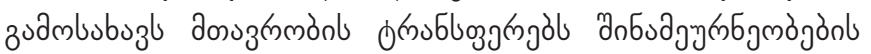

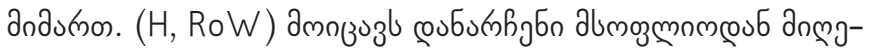

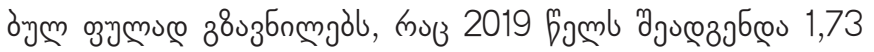

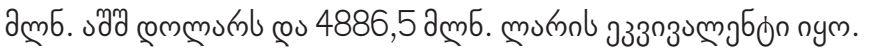

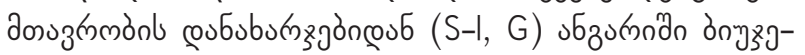

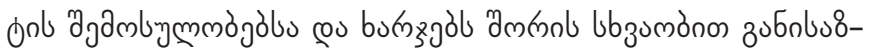

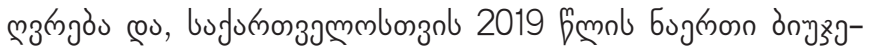

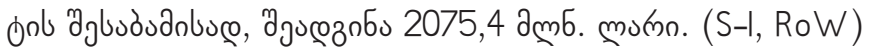

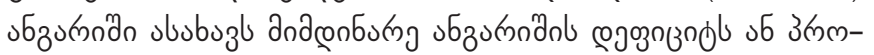

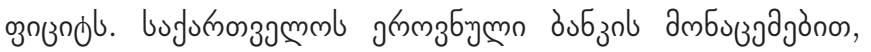

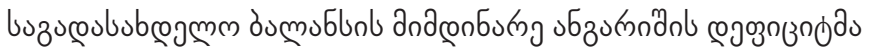

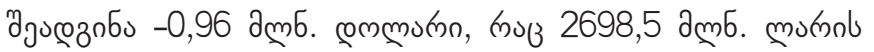

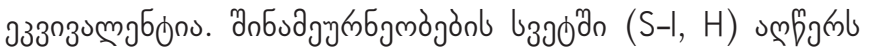

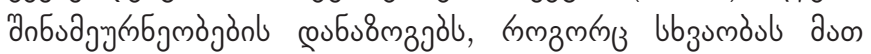

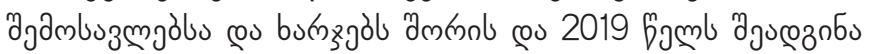
7686, 4 amb. mumn.

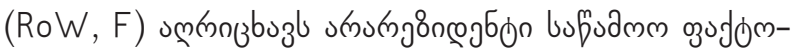

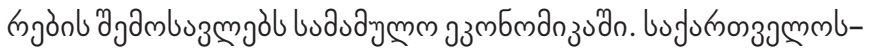
пзй

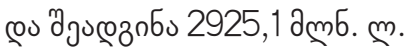

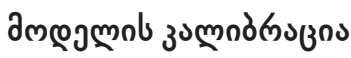

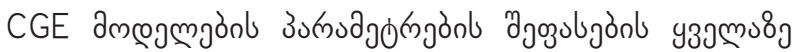

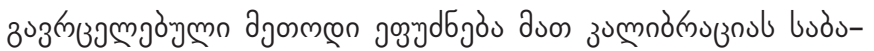
8nbm f̣mol dnbgeznon (Lofgren et al., 2002, Wing, 2003).

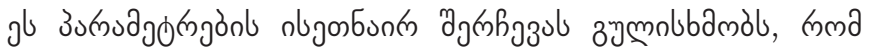

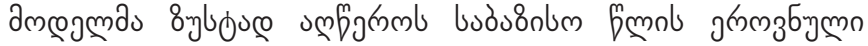

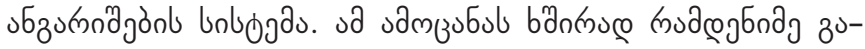

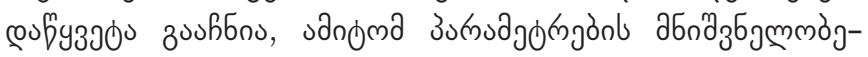

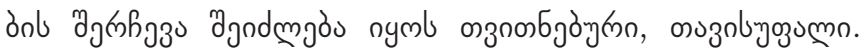

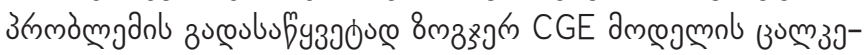

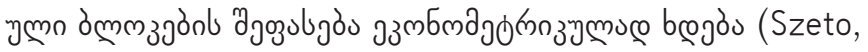

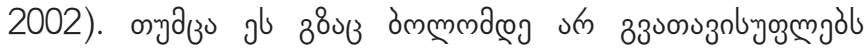

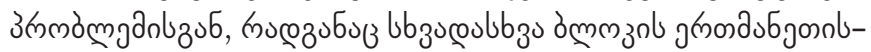

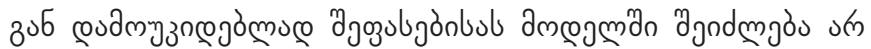

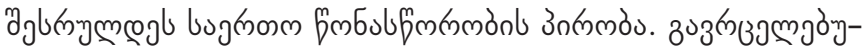

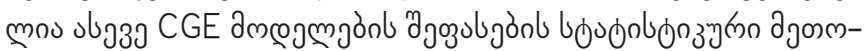

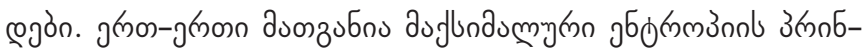

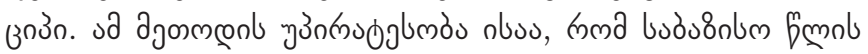

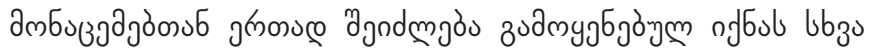

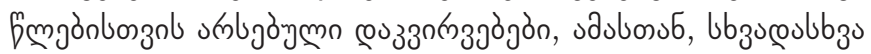

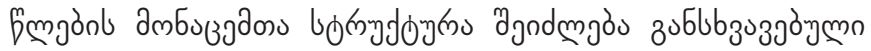
nymb.

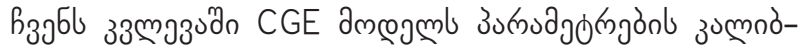

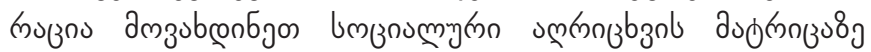

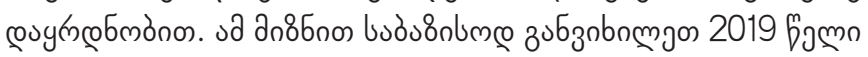

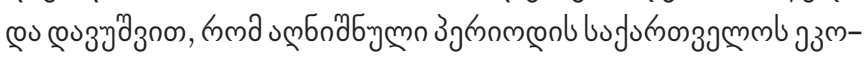

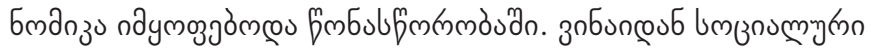

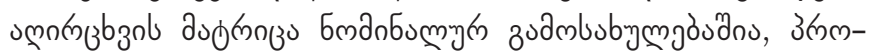

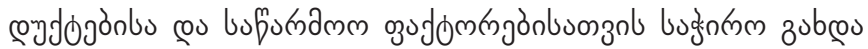

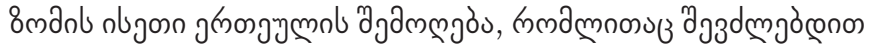

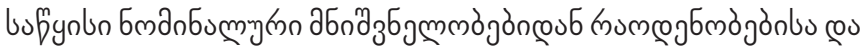

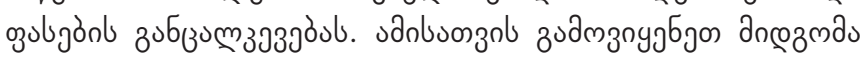

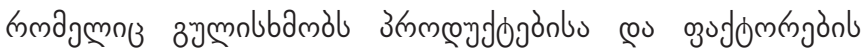

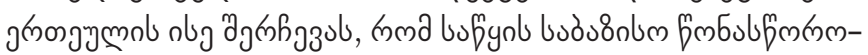
budn duon ojuln grnonl jmmn nymb (Gorbachuk \& Rusanov,

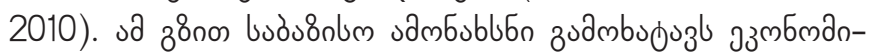
з

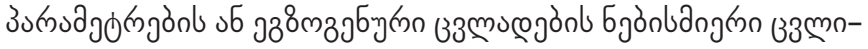

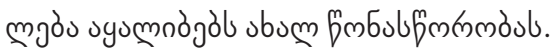

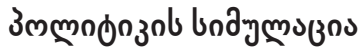

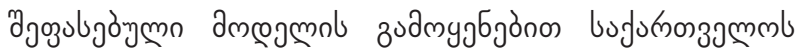

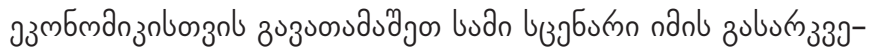

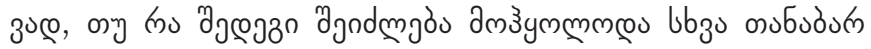

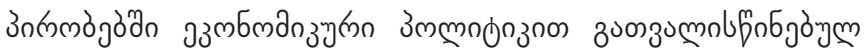

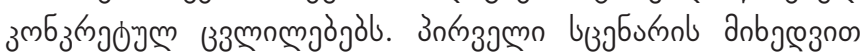

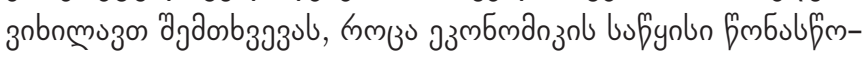

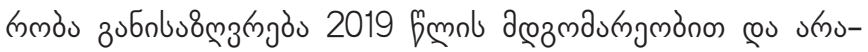

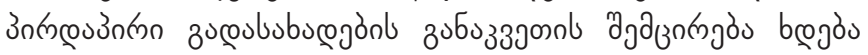

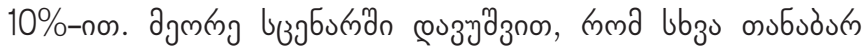

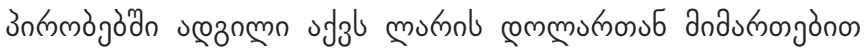

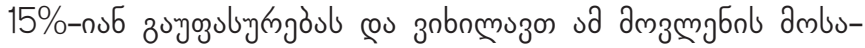

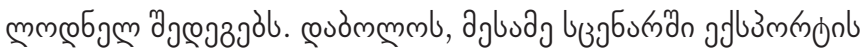




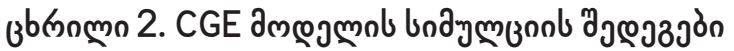

\begin{tabular}{|c|c|c|c|c|}
\hline & \multirow{2}{*}{ 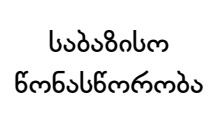 } & \multicolumn{3}{|c|}{ 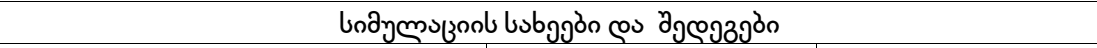 } \\
\hline & & 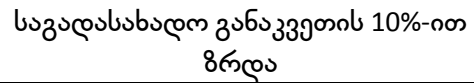 & 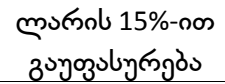 & $\begin{array}{l}\text { 9गुlummont 20\%-nos } \\
\text { 8mp̧os }\end{array}$ \\
\hline \multicolumn{5}{|c|}{ 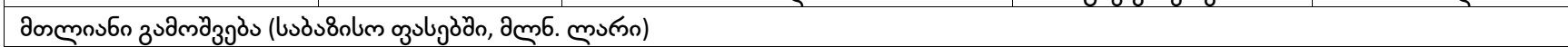 } \\
\hline 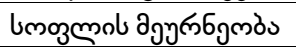 & 5176,655 & $26 \%$ & $31 \%$ & $18 \%$ \\
\hline amg6 39 mmds & 17784,734 & $-11 \%$ & $-2 \%$ & $28 \%$ \\
\hline 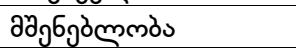 & 9866,763 & $8 \%$ & $5 \%$ & $9 \%$ \\
\hline $\begin{array}{l}\text { amabsby mgàn } \\
\text { bjdommo }\end{array}$ & 45734,707 & $-2 \%$ & $-4 \%$ & $-9 \%$ \\
\hline byen & 62562,8 & $-0,1 \%$ & $0,2 \%$ & $3,5 \%$ \\
\hline \multicolumn{5}{|c|}{ 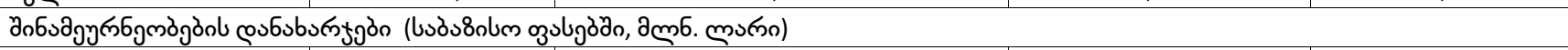 } \\
\hline lmozmol agy & 2098.068 & $17 \%$ & $13 \%$ & $15 \%$ \\
\hline дmg6 & 18543.625 & $13 \%$ & $13 \%$ & $30 \%$ \\
\hline 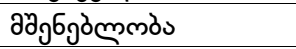 & 865.470 & $13 \%$ & $4 \%$ & $11 \%$ \\
\hline 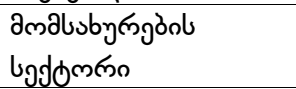 & 13241.639 & $11 \%$ & $-29 \%$ & $-26 \%$ \\
\hline byen & 34748.8020 & $12 \%$ & $-3 \%$ & $7 \%$ \\
\hline \multicolumn{5}{|c|}{ 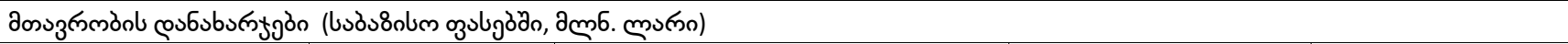 } \\
\hline \multicolumn{5}{|c|}{ 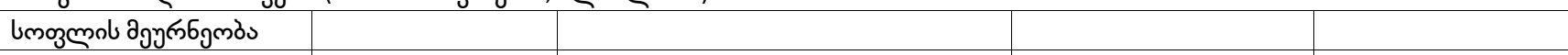 } \\
\hline anj639mmds & 76.942 & $60 \%$ & $58 \%$ & $96 \%$ \\
\hline 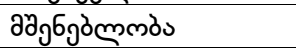 & 8.742 & $46 \%$ & $46 \%$ & $68 \%$ \\
\hline 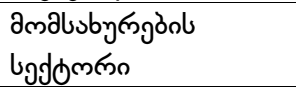 & 6371.985 & $43 \%$ & $-0,0 \%$ & $12 \%$ \\
\hline byer & 6457.6690 & $43 \%$ & $1 \%$ & $13 \%$ \\
\hline \multicolumn{5}{|c|}{ 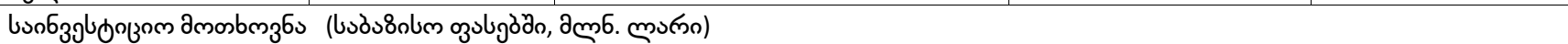 } \\
\hline lmogmol agynбggmòs & 538.834 & $11 \%$ & $14 \%$ & $13 \%$ \\
\hline 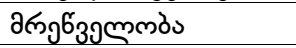 & 4193.367 & $18 \%$ & $14 \%$ & $27 \%$ \\
\hline 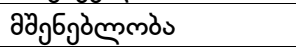 & 7497.456 & $7 \%$ & $5 \%$ & $9 \%$ \\
\hline 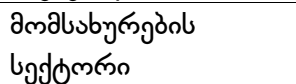 & 230.736 & $5 \%$ & $-28 \%$ & $-27 \%$ \\
\hline byen & 12460.3930 & $11 \%$ & $8 \%$ & $15 \%$ \\
\hline \multicolumn{5}{|c|}{ 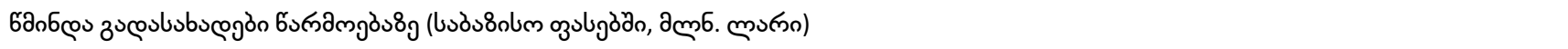 } \\
\hline lmogmol agy & -16.320 & $7 \%$ & $28 \%$ & $13 \%$ \\
\hline argo6igmmòs & 4649.819 & $-28 \%$ & $-4 \%$ & $17 \%$ \\
\hline 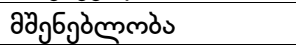 & 364.091 & $-12 \%$ & $3 \%$ & $1 \%$ \\
\hline $\begin{array}{l}\text { amabsby mgàn } \\
\text { bjdommo }\end{array}$ & 1049.319 & $-16 \%$ & $2 \%$ & $-7 \%$ \\
\hline byen & 6046.909 & $-25 \%$ & $-2,4 \%$ & $12 \%$ \\
\hline
\end{tabular}

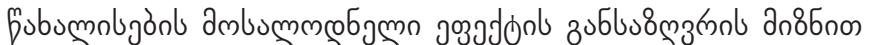

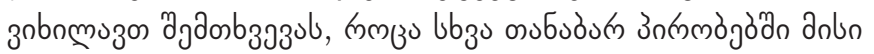

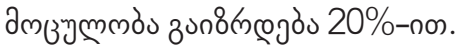

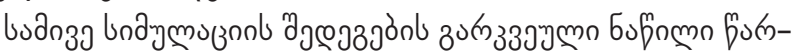

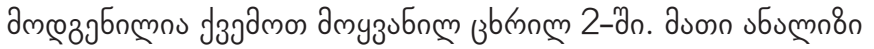

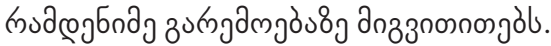

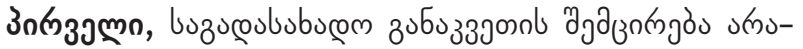

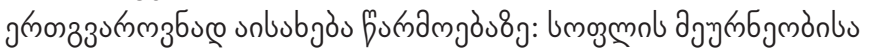

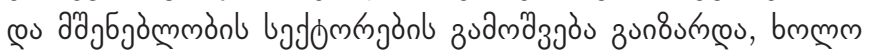

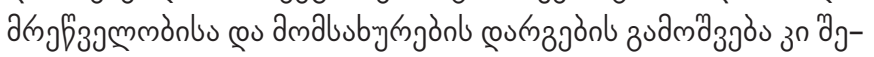

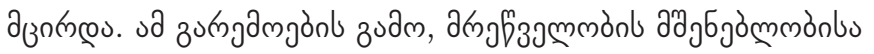

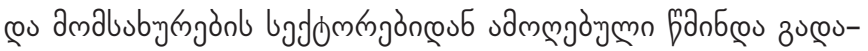

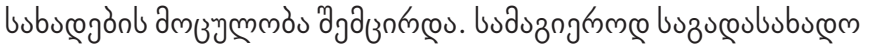

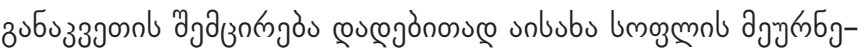

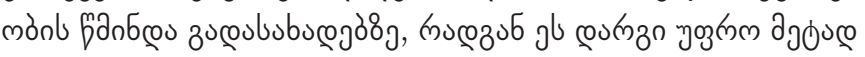

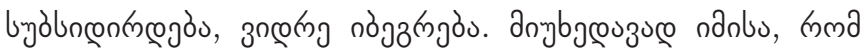

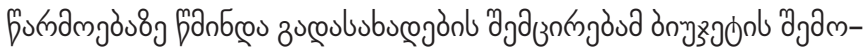

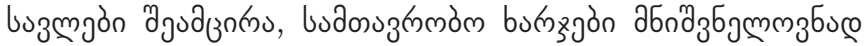

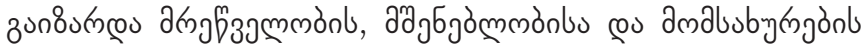

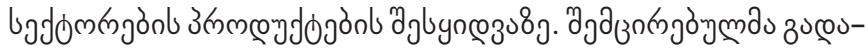
bubuल

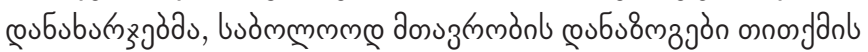
ajbuagenon a guazomo.

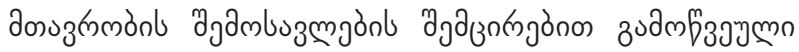

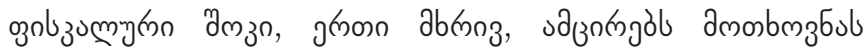

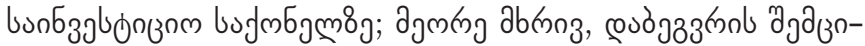




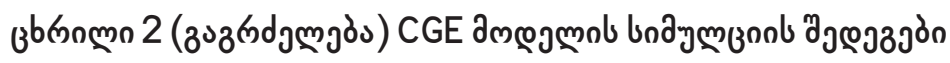

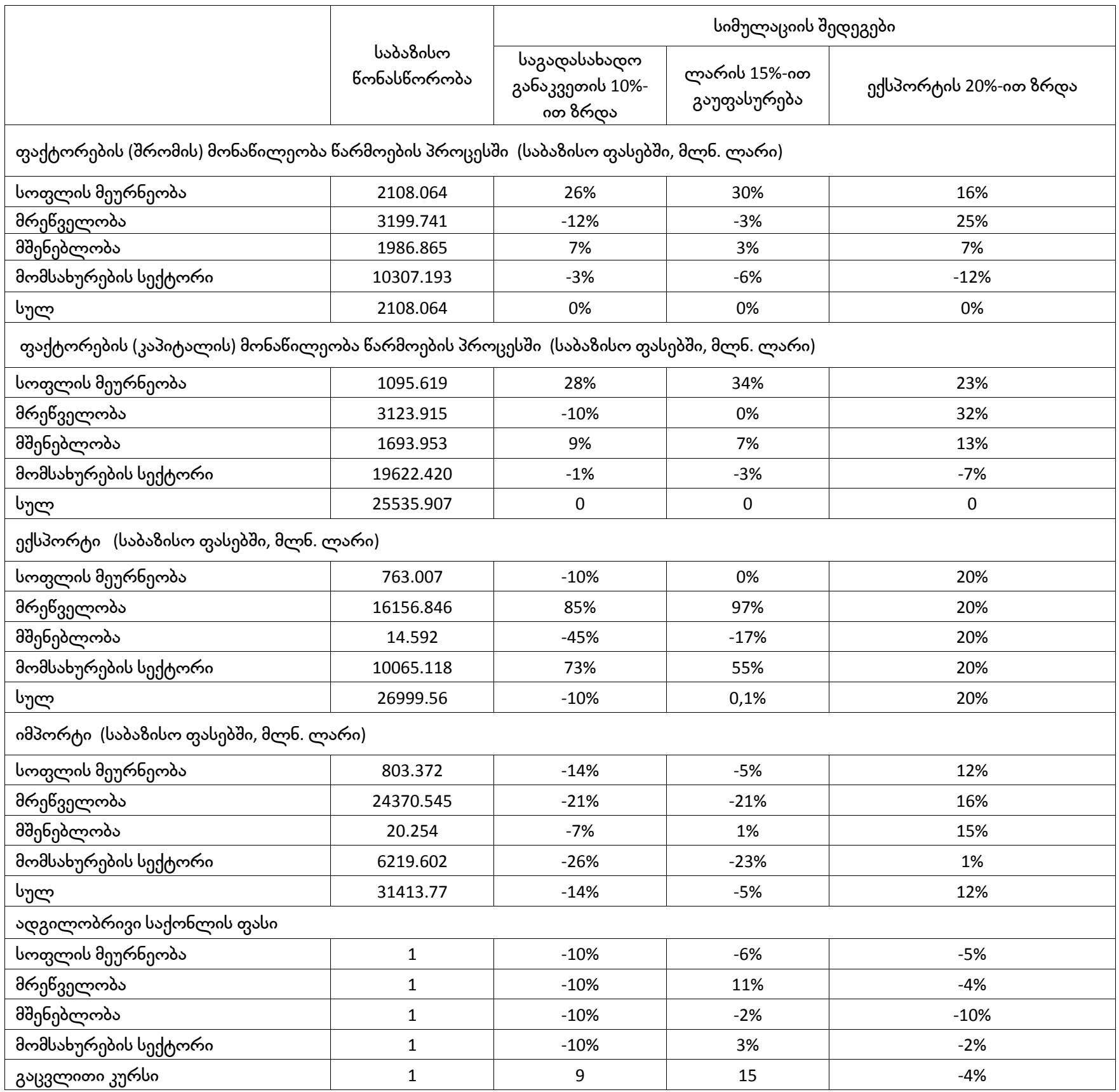

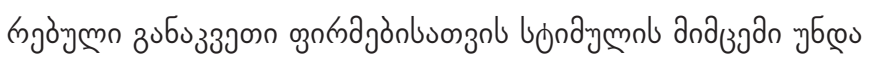

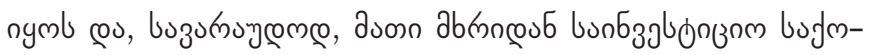

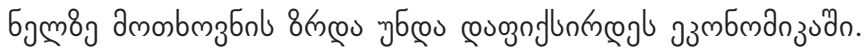

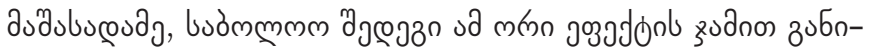

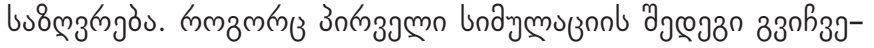

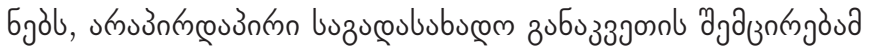

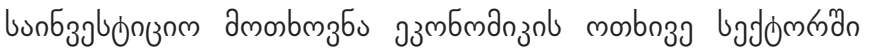

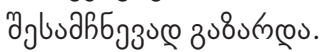

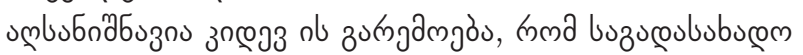

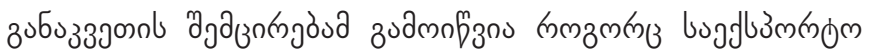

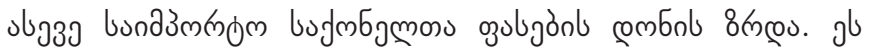

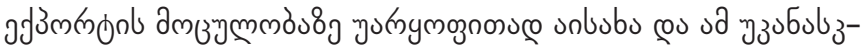

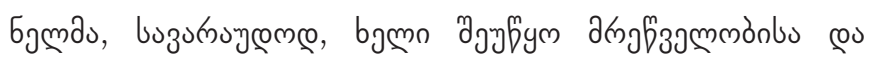

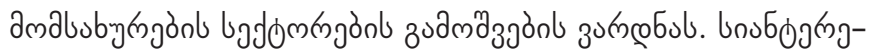

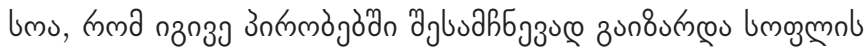

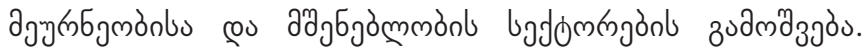

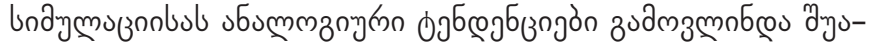

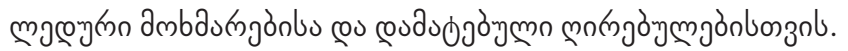

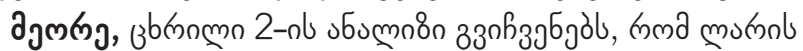

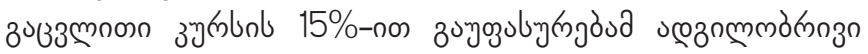

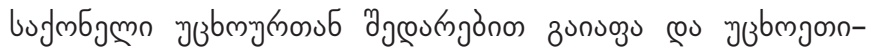

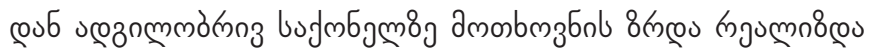

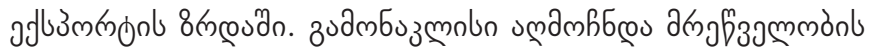

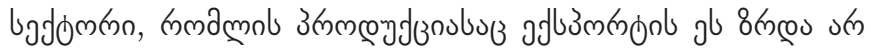




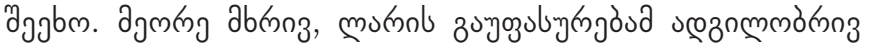

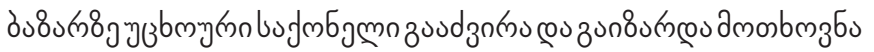
umoुmnl a agh

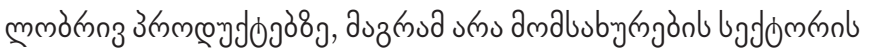

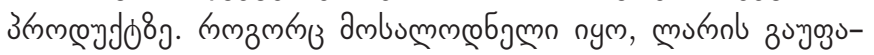

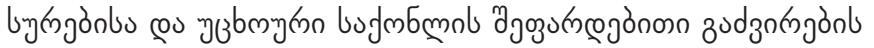

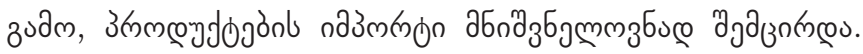

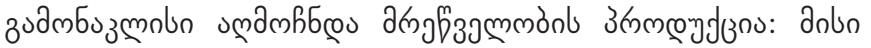

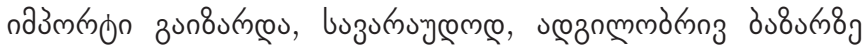

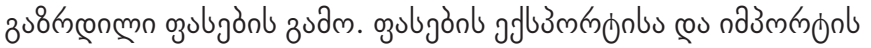

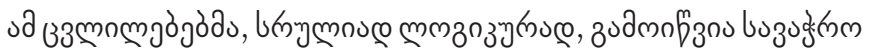

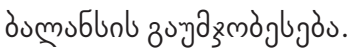

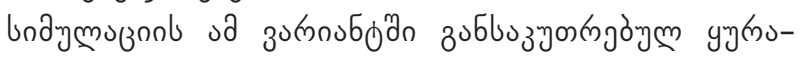

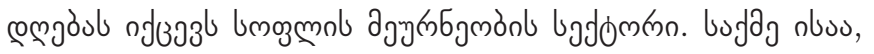

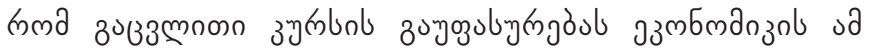

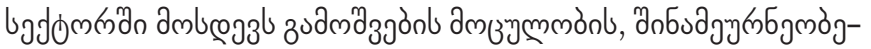

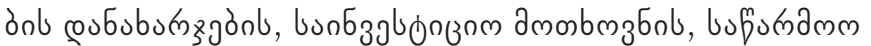

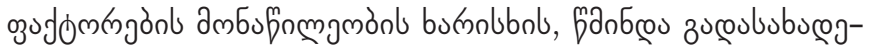

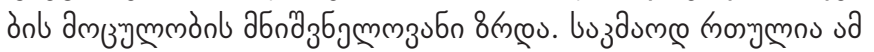

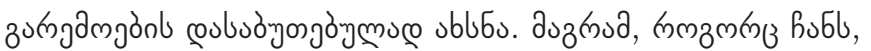

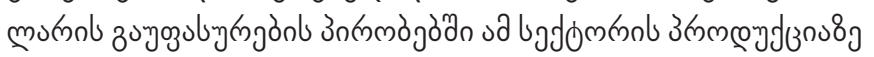

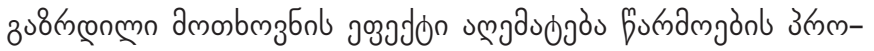

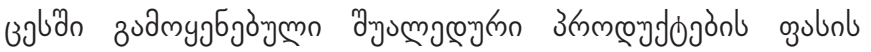
8 monl yutrymognon josadd

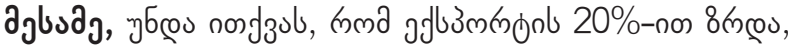

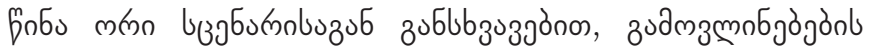

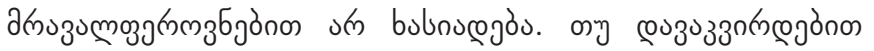

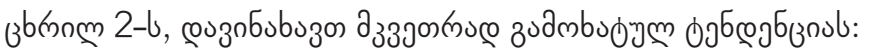

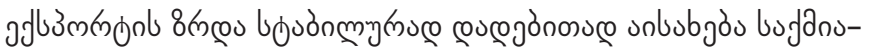

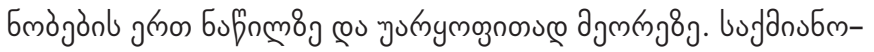

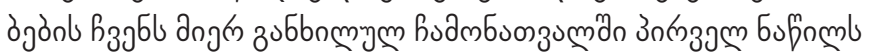

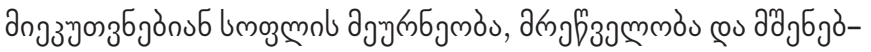

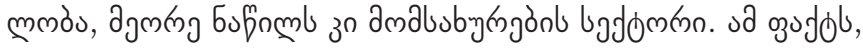

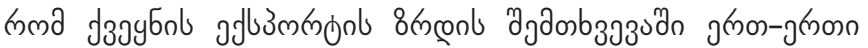

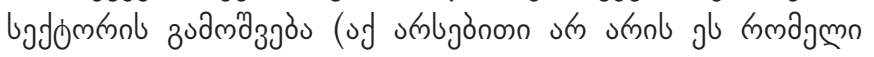

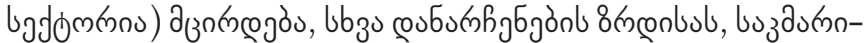

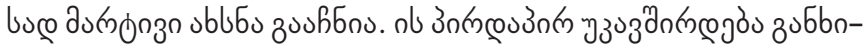

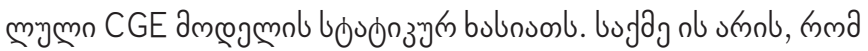

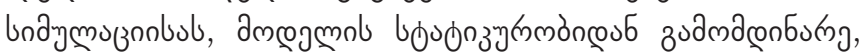

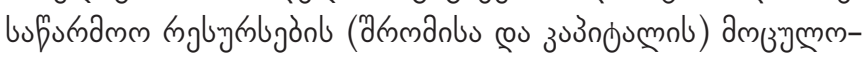

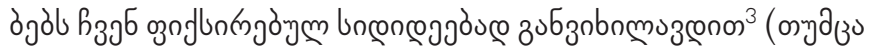

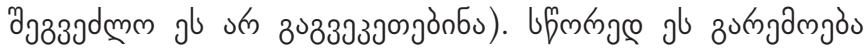

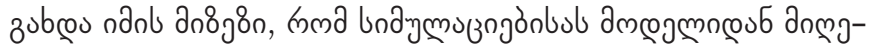

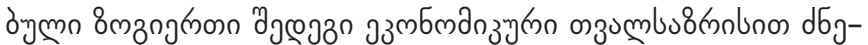

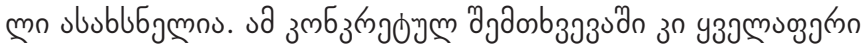

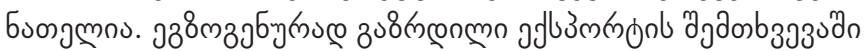

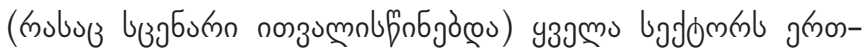

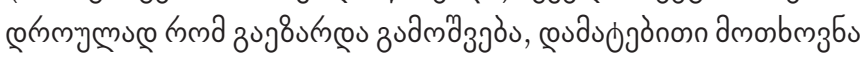

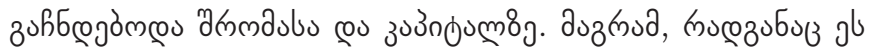

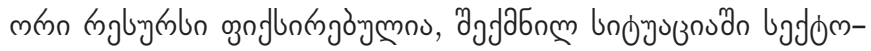
r.

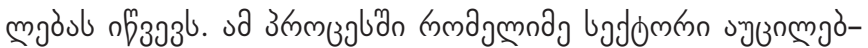

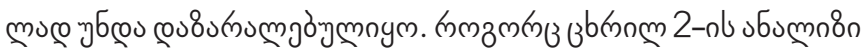

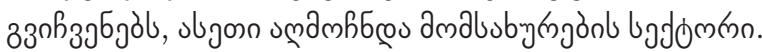

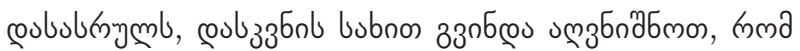

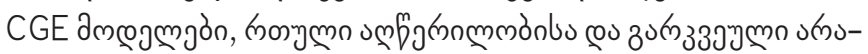

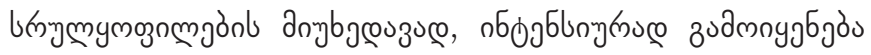

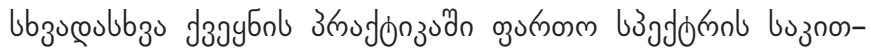

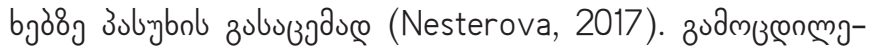

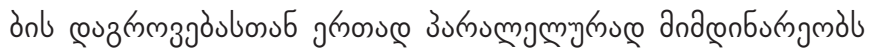

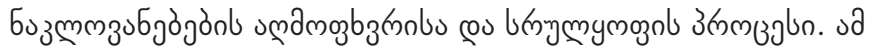

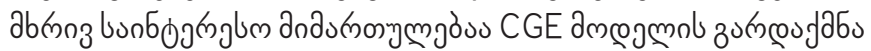

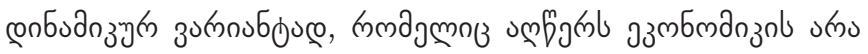

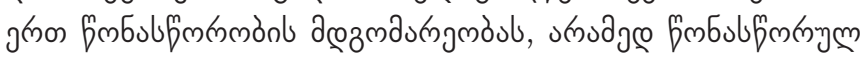

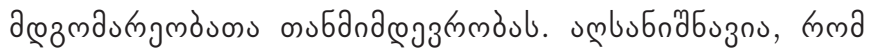

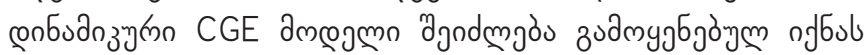

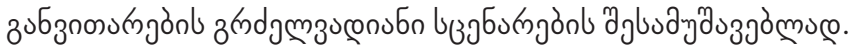

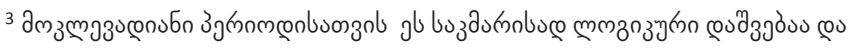

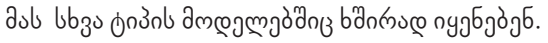

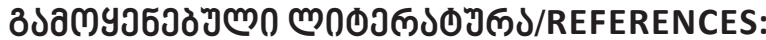

Ananiashvili, I. (2019). Monetary Politics in Quasi-linear Macroeconomic model. The 4th International Scientific Conference materials: Challenges of Globalization in Economics and Business. Tbilisi (In Georgian).

Ananiashvili, I., Achelashvili K., Meskhia I., Papava V., Silagadze A., \& Tsereteli G. (2003). Methods and Models of Macroeconomic Regulation. Tbilisi, Metsniereba (In Georgian).

Armington, P. (1969). A Theory of Demand for Products Distinguished by Place of Production. IMF Staff Papers.

Bovenberg, A. A. (1996). Optimal Environmental Taxation in the Presence of Other Taxes: General- Equilibrium Analysess. American Economic Review, 86(4), 985-1000.

Debreu, G. (1959). Theory of Value. New Haven and London: Yele Universin Press.

Decaluwe, B. A. (1999). Poverty Analysis Within a CREFA Working Paper 99-106.

Dervis, K., De Melo, J., \& Robinson, S. (1982). General Equilibrium Models for Development Policy. Washington: World Bank.

Ergeshidze, A. (2017). Impact of Exchange Rate on Macroeconomic Environment. TSU, PhD thesis (In Georgian).

Gorbachuk, V., \& Rusanov M., I. (2010). Calibration of a Computable General Equilibrium Model. Computer Mathematics. 1, 10-17 (In Russian). 
Goulder, L. (2002). Environmental Policy Making in Economies With Prior Tax Distortions. Northampton MA: Edward Elgar. Graham Pyatt, J. I. (1985). Social Accounting Matrices: a Basis for Planning. Washington: The World Bank.

Gunning, J. W., \& Keyzer, M. A. (1995). Applied General Equilibrium Models for Policy Analysis. In T. S. Hollis Chenery, Handbook of Development Economics, Edition 1. 3(35), 2025-2107.

Hosoe, N. (2004). Computable General Equilibrium Modeling with GAMS. Tokyo: National Graduate Institute for Policy Studies.

Hosoe, N., Kenji, G., \& Hideo, H. (2010). Textbook of Computable General Equilibrium Modelling: Programming and Simulations. Palgrave Macmillan.

Johansen, L. (1960). A Multi-Sectoral Study of Economic Growth. North-Holland, Amsterdam.

Labadze, L. (2015). Assessing the Effectiveness of Investments in Agriculture of Georgia through Microeconomic Model. TSU, PhD thesis ((In Georgian).

Lavrentev, A. S., \& Krinichanskii, K. V. (2016). Methods for Assessing the Impact of Structural Policy on Macroeconomic Parameters: General Equilibrium Models. Regional Economics: Theory and Practice, 9, 98-112 (In Russian).

Leontief, W. (1936). Quantitative Input-Output Relations in the Economic System of the United States.

Lofgren, H., Harris, R. L., \& Robinson, S. (2002). A Standard Computable General Equilibrium (CGE) Model in GAMS. International Food Policy Research Institute. https://www.ifpri.org/publication/standard-computable-general-equilibrium-cge-model-gams-0

Makarov, V.L, Bakhtizin A. R., \& Sulashkin S. S. (2007). Application of Computable Models in Public Administration. Nauchni Ekspert. (In Russian).

Martin, W., \& Winters, L. (1996). The Uruguay Round and the Developing Economies. Cambridge University Press: New York.

Melitauri, N. (2021). International Trade in Theoretical and Empirical Models of Macroeconomic Modelling (on the Example of Trade between European Union and Georgia). TSU, PhD thesis. (In Georgian.)

Nesterova, K. V. (2017). Multiregional Models of General Equilibrium: Framework and Applications. Upravlencheskoe Konsultirovanie. 12, 92-100 (In Russian).

Palatnik, R. R., \& Shechter, M. (2010). Assessing the Economic Impacts of Climate Change Using a CGE Model with Decentralized Market Instruments. Journal of Siberian Federal University. Humanities \& Social Sciences, 6.

Perry, G. J. (2003). Fiscal Reform and Structural Change in Developing Countries. New-York: Palgrave-Macmillan.

Rausch, S., Metcalf, G. E., Reilly, J. M., \& Paltsev, S. (2009) Policy Analysis', The MIT Joint Program on the Science and Policy of Global Change, Technical note, 6 .

Shields, C., \& François, J. (1994). Modeling Trade Policy: Applied General Equilibrium Assessments of North American Free Trade. New York: Cambridge University Press.

Stone, R. (1947). Measurement of National Income and the Construction of Social Accounts. Geneva: United Nations.

Stone, R. (1962). A computable Model of Economic Growth. London: Chapman and Hall.

Szeto, K. L. (2002, June 2). A Dynamic Computable General Equilibrium (CGE) Model of the New Zealand Economy. New Zealand Treasury Working Paper. http://nzae.org.nz/files/\%2363-SZETO.PDF.

Thorbecke, E. G. (1976). Planning Techniques for a Better Future. Geneva.

Weyant, J. (1999). The Costs of the Kyoto Protocol: a Multi-Model Evaluation. Energy Journal (special issue).

Wing, S. I. (2004). Computable General Equilibrium Models and their Use in Economy-Wide policy Analysis. 